\title{
Synaptic scaling rule preserves excitatory/inhibitory balance and salient neuronal network dynamics
}

Jérémie Barral ${ }^{1}$, and Alex Reyes ${ }^{1}$

${ }^{1}$ Center for Neural Science, New York University, New York, USA

Correspondence should be addressed to J. B. (barral@cns.nyu.edu).

\section{Summary}

The balance between excitation and inhibition ( $E-I$ balance) is maintained across brain regions though the network size, strength and number of synaptic connections, and connection architecture may vary substantially. Here, we use a culture preparation to examine the homeostatic synaptic scaling rules that produce $E$ - $I$ balance and in vivo-like activity. We show that synaptic strength scales with the number of connections $(K)$ as $\sim 1 / \sqrt{K}$, close to the ideal theoretical value. Using optogenetic techniques, we deliver spatiotemporally patterned stimuli to neurons and confirm key theoretical predictions: $E-I$ balance is maintained, active decorrelation occurs, and the spiking correlation increases with firing rate. Moreover, the trial-to-trial response variability decreased during stimulation, as observed in vivo. These results - obtained in generic cultures, predicted by theory, and observed in the intact brain - suggest that the synaptic scaling rule and resultant dynamics are emergent properties of networks in general.

\section{Introduction}

The firing dynamics of neural networks depend on the overall balance between excitation $(E)$ and inhibition $(I)$. Maintaining a balance of $E$ and $I$ inputs to neurons is crucial for coding ${ }^{1}$ and severe imbalances can lead to neuropathologies ${ }^{2-6}$. The balanced state, characterized by neuronal activities that are neither completely silent nor saturated ${ }^{7,8}$, exists in a wide range of networks with different configurations. The size, connection architecture, synaptic dynamics and strength, and intrinsic properties of $E$ and $I$ neurons vary widely across brain regions ${ }^{9-11}$ and may change during development ${ }^{12-15}$ and learning ${ }^{16}$. The presence of the balanced state under these different conditions suggests that potentially many network variables are adjusted homeostatically to maintain $E-I$ balance and functional network dynamics. 
Determining the homeostatic rules that lead to balance is difficult given the complexity of cortical circuits. One approach is to reduce the number of variables and use well-established mean field techniques adapted from statistical mechanics to examine general network properties analytically ${ }^{7,8,17}$. Rather than incorporating as many of the experimentallydetermined variables as possible ${ }^{18}$, only a few key parameters of the network are considered: the number $(N)$ of excitatory and inhibitory neurons; the strength $(J)$ and number $(K)$ of synaptic connections per neuron; and the connection probability $\left(P_{c}\right)$ between cells. For mathematical rigor, mean field theories are developed in the limit of infinite network size and assume statistical independence between the variables. The network behaviour depends critically on how $J, K, P_{c}$, and $N$ scale with respect to each other. Expressions for the mean and variance of the network-generated synaptic input and conditions to achieve $E-I$ balance can be derived readily (see Supplementary Text). In this framework, general properties such as the firing rate or the correlation between neurons can be analytically predicted from network properties ${ }^{7,8,17}$. Ideally, any scaling scheme should not only achieve balance but also reproduce the salient features of in vivo activity and be constrained by experimentallydetermined parameters.

Under the constraint that the highly irregular firing of in vivo neurons is preserved ${ }^{19-21}$, one scheme $^{7,8}$ requires that $J$ scales with the number of connections $K$ as $1 / \sqrt{K}$. This scaling ensures that the fluctuations or variance $\left(\sigma^{2}\right)$ in the synaptic input ( $\sigma^{2}$ is proportional to $K$. $J^{2}$, see Supplementary Text) does not depend on the number of connections ( $\sigma^{2}$ proportional to $K \cdot(1 / \sqrt{K})^{2}=$ constant $)$. Fluctuations cause stochastic crossings of threshold and hence irregular firing. Note that if instead $J$ scales as $1 / K$, the variance vanishes with increasing $K$. The balanced state is achieved provided that the mean excitatory synaptic input $E$ (composed of recurrent and external drive to the network) is equal in magnitude to the mean inhibitory synaptic input $I$ so that the composite synaptic input $(E-I)$ is close to rheobase (see Supplementary Text).

Another constraint is that correlation in the firing of neurons is low. Low correlation is crucial for ensuring statistical independence of variables required for mean field ${ }^{7,8,17}$ and for efficient coding of firing rate information under some circumstances ${ }^{22}$. To maintain low spiking correlations, one possibility is to set $K$ to be large but much smaller than $N$ $(1 \ll K \ll N)$ by e.g. defining $K$ to be a constant or to increase at a very slow rate with $N$. Under this condition, the probability $P_{c}(=K / N)$ that neurons are connected is low ('sparsely-connected' network ${ }^{7,8,17}$ ); correlations are reduced because the probability that two 
neurons receive common inputs $\left(=P_{c}{ }^{2}\right)$ is also small. However, estimates of the connection density of local cortical networks suggest that wiring is not sparse, especially when considering excitatory-inhibitory wiring ${ }^{14,18,23-29}$. Nevertheless, correlations can also be small in networks where $K$ increases proportionately with $N$ so that $P_{c}$ is non-vanishing ('denselyconnected' network). Although dense connectivity induces positive correlations between isolated $E$ inputs and between isolated $I$ inputs to neurons, correlations in the composite synaptic input and spiking is reduced because the $E$ and $I$ inputs co-vary in time (track each other) and cancel. Moreover, the correlation is predicted to decrease with network size ${ }^{30}$. In addition to network parameters, spiking correlation is also affected by correlations in the external drive to the network ${ }^{30}$ and by the firing rate of neurons ${ }^{31}$.

A shortcoming of the reduced approach is that many of the simplifying assumptions needed for mathematical tractability appear far from physiological parameters. The $1 / \sqrt{K}$ scaling rule and the relations between the variables were derived based not on biological principles but rather on the fact that the model reproduced in vivo-like activity. Furthermore, the idealized network differs substantially from biological networks, in which $K$ and $N$ are finite, neurons have diverse membrane and firing properties, $P_{c}$ varies with distance and cell type, and $J$ exhibits time-dependent depression or facilitation.

Here, we use a culture preparation where $N$ can be systematically varied and $K, J$, and $P_{c}$ can be measured accurately. To determine whether the scaling results in $E-I$ balance, high spiking variability, and low correlations, we drive the network with specified spatiotemporal patterns using optogenetic stimulation. Using a variety of stimuli delivered under various conditions, we test whether the results hold under conditions different from the ideal theoretical limits and whether the network can accommodate other network behaviours not explicitly predicted by the theories.

\section{Results}

\section{Intrinsic and network properties of cortical cultures}

To confirm that the properties of cortical neurons grown in the glia-free cultures were similar to those of neurons in acute slices, we characterized the intrinsic and synaptic properties of neurons by performing paired whole-cell recordings $(n=491$ cells, 1080 tested connections in 131 preparations). A neuron was classified as excitatory or inhibitory based on whether suprathreshold stimulation evoked depolarizing or hyperpolarizing responses in its 
postsynaptic target (Fig. 1a). Approximately $23 \%$ of the neurons in culture were inhibitory, consistent with estimates in cortical slices ${ }^{27}$ and in cultures ${ }^{32,33}$. By 14 days in vitro (DIV), the resting membrane potential, the input resistance and the membrane capacitance remained constant for at least 30 DIV (Supplementary Fig. 1, Supplementary Table 1) and were similar to those measured in cortical slices ${ }^{15,24,27}$. The density did not change significantly with DIV for the range examined (Supplementary Fig. 1) ${ }^{33}$.

As in in vitro slices of cortex ${ }^{14,24}$, the connection probability $\left(P_{c}\right)$ between neurons decreased with distance, following a Gaussian profile (Fig. 1b). Organization principles seemed similar to those measured in cortical slices $\left(p_{E \rightarrow E} \sim 0.2, \quad p_{E \rightarrow I}=0.3-0.6, \quad p_{I \rightarrow E}=0.4-0.7\right.$, and $p_{I \rightarrow I}=0.3-0.7$ for reported values ${ }^{14,18,23-29}$ ) with lower peak probability between $E$ cells $\left(p_{E \rightarrow E}=0.38\right)$ than between $E$ and $I\left(p_{E \rightarrow I}=0.59\right.$ and $\left.p_{I \rightarrow E}=0.64\right)$ or between $I$ cells $\left(p_{I \rightarrow I}=0.59\right)$ (Supplementary Fig. 2, Supplementary Table 2). However, the spatial profiles were wider in this 2-dimensional network than those in slices. The connection probability across all cell types decreased with a characteristic length of about $600 \mu \mathrm{m}$ as compared to $100-200 \mu \mathrm{m}$ in cortical slices ${ }^{24,25,28,29}$.

To examine how the profile of connections varied with network size, we pooled data according to densities. The area under the connection profile $P_{c}$ did not change substantially with density (Fig. 1b-c, Supplementary Fig. 2g). For each density, we estimated that the total number $(E+I$; black Fig. 1c) of presynaptic inputs $(K)$ to a neuron by integrating the probability profiles over space (see Methods and inset of Fig. 1c), which provided a calibration curve relating the expected number of connections as a function of density. This allowed us to determine that $K$ increased linearly with density $D$ (Fig. 1c, fit: $K=0.82 \cdot D$, $\left.R^{2}=0.995\right)$ indicating that cortical neurons in culture form densely-connected networks.

\section{Scaling of synaptic strength with network size}

The amplitudes of excitatory and inhibitory postsynaptic potentials (EPSPs and IPSPs) decreased with density (Fig. 1a). At low densities $\left(<100\right.$ neurons $\left./ \mathrm{mm}^{2}\right)$, the unitary EPSP and IPSP amplitudes were $4.3 \pm 1.4 \mathrm{mV}$ and $-3.8 \pm 1.7 \mathrm{mV}$ (mean $\pm \mathrm{SD})$, respectively, while at high densities (>300 neurons $/ \mathrm{mm}^{2}$ ), the values were $1.3 \pm 1.1 \mathrm{mV}$ and $-1.4 \pm 1.6 \mathrm{mV}$, consistent with previous results ${ }^{34,35}$. Using a general linear model, we determined that the amplitudes did not vary significantly with neuron type, DIV, distance, or intrinsic properties (Supplementary Table 3). 
Plotting the PSP amplitudes vs the expected number of connections $K$ shows that the strengths $J$ of both $E$ and $I$ synapses scale with $K$ with an inverse power law (Fig. 1d). Fitting the combined $E-I$ data in log-log scale (PSP amplitudes are lognormally distributed, Supplementary Fig. 3) gives an exponent of -0.59 (95\% confidence interval: [-0.70:-0.47], $R^{2}=0.232$ ), comparable to the theoretically proposed $K^{-0.5} \mathrm{scaling}^{7,8}$. Individual fits for unitary EPSPs and IPSPs have exponents of -0.60 and -0.52 , respectively. Assuming that there are 1500 connections per neuron in the intact cortex ${ }^{18}$, this scaling $\left(J(\mathrm{mV})=32 \cdot K^{-0.59}\right)$ predicts a PSP amplitude of $\sim 0.4 \mathrm{mV}$, well within an order of magnitude of the unitary PSP amplitudes $(\sim 0.1-0.5 \mathrm{mV})$ measured in cortical slices ${ }^{14,18,24,28,36}$.

\section{Spiking dynamics of optogenetically-driven network}

To examine the spiking dynamics in the activated network, we stimulated a subset of neurons that expressed channelrhodopsin (ChR2) using a Digital Light Processing projector (Fig. 2a,b). ChR2 (and a fluorescent tag) were expressed either only in $E$ cells (transgenic lines) or non-specifically in both $E$ and $I$ neurons (viral injection). Regions of interest (ROIs) covering the ChR2-expressing neurons were defined and simultaneous cell-attached or wholecell recordings established in 4 cells that either did not express ChR2 or whose somata did not overlap with the processes of neurons in the ROI (Fig. 2a). Stimuli were trains of random light pulses with input rates $v_{\text {stim }}$ ranging from 2-40 Hz (see Methods, Supplementary Fig. 4). Because the light pulses were suprathreshold (Supplementary Fig. 5), the stimulated neurons approximate the external feedforward input to the network (Fig. 2c), though feedback from the network may generate additional, albeit weaker, activity in the stimulated neurons (Supplementary Fig. 5). Because the ChR2-expressing neurons were often close and had overlapping processes, each ROI may activate 1-4 neurons. Experiments in which ChR2 was expressed sparsely so that neurons could be stimulated individually, produced qualitatively similar results (Supplementary Fig. 6). Note that spontaneous, network-wide bursts present in cultures were excluded from analyses (see Data Analyses in the Methods section for rationale).

The responses in simultaneously recorded neurons in cell-attached (extracellular) mode were heterogeneous. Asynchronous stimulation of each ROI at $5 \mathrm{~Hz}$ increased firing rate above baseline activity in approximately $65 \%$ of non-stimulated neurons (106 out of 164 neurons in 44 cultures; see Fig. 3a,b). Identical stimuli delivered to the network evoked robust activity in some (cells 1 and 3 in Fig. 3a) and little in others (cell 2 and a $4^{\text {th }}$ cell that did not fire; see also Supplementary Figs. 6 and 7). Such variability likely occurred because, a finite number 
of neurons within an enclosed area were stimulated so that local heterogeneity in the network circuitry produced differences in the average $E$ and $I$ inputs to each cell.

The evoked activity is consistent with several key theoretical predictions. First, the action potential firing were irregular: the Fano factor was $\sim 1$ and did not increase with the observation window (Supplementary Fig. 8a), indicating a near Poisson process. Second, in accordance with the $1 / \sqrt{K}$ scaling $^{7,8}$, the spiking variability did not decrease with increasing $N$ (Fig. 3c, red). Third, the distribution of firing rates was long tailed and approximately lognormal (Fig. 3b and Supplementary Fig. 8b-c), which is predicted by balance network theory $^{7,8,37}$ and is also in line with experimental results ${ }^{38}$.

In addition, the network exhibit firing behaviours that are consistent with those documented in intact animals with natural stimuli. As observed in vivo ${ }^{39}$ and predicted by theory ${ }^{40}$, stimulation of the network quenches variability in the number of spikes evoked over a time interval (Fig. 3c). The increase in firing rate during stimulation (Fig. 3b) was accompanied by a significant drop in the Fano factor across all densities (Fig. 3c). Examination of evoked activity during several sweeps of identical stimuli shows that the times of occurrences of some spikes were repeatable across trials (Fig. 3a, see also Supplementary Figs. 6 and 7). To characterize the underlying synaptic inputs, whole-cell recordings with $\mathrm{Na}^{+}$channels blocker QX-314 present in the internal solution, were then established in the same cells (shown for cell 3; Fig. 3a, see also Supplementary Fig. 7). Large voltage transients coincided with the reliable extracellularly recorded spikes while variable membrane potentials coincided with the unreliable spikes. Other spikes were variable over repetitions of the identical stimulus and likely resulted from variability generated by the recurrent activity.

\section{$E-I$ balance in the activated network}

To confirm that the $E$ and $I$ synaptic potentials evoked during stimulation are balanced, whole-cell current clamp recordings were established in 1-4 cells (Fig. 4a). At resting potential, the evoked compound PSP (cPSP) was a mixture of EPSPs and IPSPs. To isolate the EPSPs (IPSPs), the membrane potential was held at $-80 \mathrm{mV}(0 \mathrm{mV})$, near the reversal potential for IPSPs (EPSPs) and stimuli identical to that used to evoke cPSPs were delivered. Consistent with the balanced regime in in vivo data ${ }^{41}$, the EPSPs (red in Fig. 4a) were countered by IPSPs (blue) of comparable amplitude, resulting in smaller cPSPs (black). Balance was achieved at the cellular level: the average evoked IPSPs and EPSPs to a single cell were linearly related (Fig. 4b) similar to in vivo findings ${ }^{42}$. 
The balanced regime was maintained irrespective of network size. The mean cPSP did not increase significantly with network density (despite the $\sqrt{K}$ term in Eq. 3 of Supplementary Text) because the excitatory drive was matched by a comparably size inhibitory drive (Fig. 4c). In accordance with the $1 / \sqrt{K}$ scaling of synaptic strength, the variability of EPSPs, IPSPs, and cPSPs during stimulation did not decrease with density (Fig. 4d). This effect was maintained for both low (Fig. 4a, left) and high (right) number of stimulated neurons.

In the balanced regime, the mean firing rate of neurons should on the average be proportional to the magnitude of the external drive $e^{7,8,30}$. Increasing the number $\left(N_{\text {stim }}\right)$ of ROIs caused a linear increase in the evoked rate ( $v$ ) (Fig. 4e) and in the average (Fig. 4a and f) and standard deviations (inset of Fig. 4f) of EPSPs, IPSPs, and cPSPs. Similar observations were made when the rate of each ROI ( $v_{\text {stim }}$; at constant $N_{\text {stim }}$ ) was increased (Fig. $4 \mathrm{~g}, \mathrm{~h}$ ). The apparent non-linear relation became more linear (Supplementary Fig. 9) when $v_{\text {stim }}$ was corrected for the frequency-dependent decline in the efficacy of ChR2 (Supplementary Fig. 5). Synaptic depression and/or firing rate adaptation may also contribute to the saturation. The firing rate gain $\left(v / v_{\text {stim }}\right)$ was homeostatically maintained and did not change with density (Figs. $3 \mathrm{~b}$ and $4 \mathrm{~g}$; the mean drive is proportional to $N_{\text {stim }} \cdot J$ and was kept constant at each density by scaling $N_{\text {stim }}$ by $\sqrt{\text { density }}$.

\section{Spiking and membrane potential correlations in driven networks}

Because the networks are densely-connected ( $K \propto N$ so that $P_{c}$ is constant) with the $K^{-0.59}$ synaptic scaling, neurons share substantial and strong inputs from common sources, which would result in significant spiking correlation in the large $N$ limit. However, correlations are reduced because $E$ and $I$ track and cancel each other ${ }^{30,41,43}$ : during stimulation, the fluctuations in the isolated $E$ inputs to neurons mirror those in $I$ inputs (Figs. $4 \mathrm{a}$ and 5a). A hallmark of $E$ $I$ tracking is that the correlation between $E$ inputs to two neurons and between isolated $I$ inputs is larger than that between cPSPs. This was confirmed in simultaneously-recorded pairs by cross-correlating the isolated EPSPs (Fig. 5a, red), isolated IPSPs (blue), and cPSPs (black). Similar results were obtained by cross-correlating the trial-averaged traces ('signal correlation') and by cross-correlating the individual traces after subtracting the averaged traces ('noise correlation') (Supplementary Fig. 10).

In the asynchronous state, the spiking correlation decreases with $N$ due to improved $E-I$ tracking $^{30}$. As predicted, increasing the network density reduced both the correlations in spikes and cPSPs while $E-E$ and $I-I$ correlations remained high (Fig. 5b). 
To confirm that $E-I$ tracking attenuates correlations in the external drive ${ }^{30}$, we systematically varied the correlation between the stimulated neurons. Correlations in the light stimuli were adjusted via the $C_{\text {stim }}$ parameter of the algorithm for generating pulse trains ${ }^{31}$ (Supplementary Fig. 4 and Methods). The time courses of both the EPSPs and IPSPs mirrored each other and changed in parallel with $C_{\text {stim }}$ (Figs. 5a, Supplementary Fig. 6) to preserve both E-I balance (Supplementary Fig. 11) and tracking. The signal (Fig. 5c) and noise (Fig. 5d) correlations between cPSPs were substantially less than those between the isolated EPSPs or IPSPs. The

noise correlation did not change with $C_{\text {stim. }}$. The increase in signal correlation with $C_{\text {stim }}$ reflects the fact that the tracking was not perfect. Because only $E$ neurons were stimulated, there was a short delay between excitation and inhibition, which prevented complete cancellation and became more apparent at high $C_{\text {stim }}$. The enhanced voltage transients in the cPSPs with increasing $C_{\text {stim }}$ (Fig. 5a, black) resulted in more precise spiking and hence high spike correlations (Fig. 5e). Using cultures where both $E$ and $I$ neurons were photo-activated simultaneously to eliminate the $E-I$ delay reduced correlations in the cPSP and therefore in the spikes (Supplementary Fig. 12).

Finally, we confirm that spiking correlation co-vary with the firing rate of the neurons due to the threshold non-linearity in the neuronal transfer functions ${ }^{31}$. This relation between rate and correlation has been difficult to examine in vivo because potential correlations in the recurrent synaptic input $C_{i n}$ cannot be controlled. As predicted, increasing stimulus rate $\left(v_{\text {stim }}\right.$, as in Fig. 4g), increased both the firing rate and spiking correlations between neuron pairs (Fig. 6a). Similar results were obtained with $v_{\text {stim }}$ fixed at $5 \mathrm{~Hz}$, which produced a sufficiently broad range of firing rates for analyses (Fig. 6b). The increase in correlation was not accompanied by an increase in $C_{i n}$ : the signal (Fig. 6c) and noise (Fig. 6d) correlations in the subthreshold membrane potential (cPSP-cPSP, black) were flat.

\section{Discussion}

In summary, the data indicate that the $1 / \sqrt{K}$ scaling rule predicted by theory occurs in a network of live neurons. This scaling ensured that $E-I$ balance and network dynamics were homeostatically maintained in different size networks. The biological mechanism underlying the scaling is unknown but may be related to the network parameters as follows. To a first approximation, $J=s_{b} \cdot n_{b}$ where $n_{b}$ is the number of boutons from a single presynaptic cell $^{18,29}$, each of which evokes a unitary response with magnitude $s_{b}$. The total number of 
boutons onto a cell is therefore $N_{b}=K \cdot n_{b}$ so that $J=s_{b} \cdot N_{b} / K$. In cultures, $s_{b}$ (taken to be miniature EPSPs) is constant ${ }^{35}$ suggesting that it is $N_{b}$ that should increase as $\sqrt{K}$ to satisfy the $1 / \sqrt{K}$ scaling.

Indeed, several lines of evidence suggest that the number of synaptic boutons per neuron increases with the number of neurons in the culture. Increasing the density 8-fold decreased synaptic strength 3 -fold but increased the number of dendritic spines 2-fold ${ }^{35}$. Similarly, increasing the network size 10-fold while maintaining constant density weakened synaptic strength 4-fold but increased the number of boutons 2-fold ${ }^{34}$. Altogether, these results suggest that the decreased synaptic strength in denser networks is mediated by a sublinear increase in the number of synaptic boutons. The underlying physiological and molecular mechanisms are unknown but may be related to the homeostatic processes that regulate activity in culture ${ }^{44}$ and in vivo ${ }^{45}$. In these cases, a combination of synaptic scaling and modification of intrinsic properties regulate the overall activity of the neuronal network in response to a stress. In our experiments, activity level and variability, both of which are constant across density, may be the set point variables.

Two concerns are that the culture preparation does not fully replicate the conditions in vivo and that the cellular and synaptic characteristics depend on the particular methodology ${ }^{46,47}$. Under the conditions of our experiments, the intrinsic properties of neurons and the projected PSP amplitudes calculated with the scaling rule are comparable to those measured in acute slices. Importantly, the evoked activity of the cultured neurons replicated several salient features of in vivo activity -irregular firing and stimulus-dependent decrease in trial-to-trial spiking variability ${ }^{39}$, lognormal distribution of firing rate $^{38}, E-I$ tracking ${ }^{41,42}$ - found in functionally diverse brain areas. Thus, cultures contain essential elements common to neuronal networks in general.

With unprecedented control of key experimental variables, we confirmed the major predictions of seminal theories and show that they hold under conditions far from the asymptotic limits where $K$ and $N$ are large. Spike variability, the balanced regime, and decorrelation by $E-I$ tracking occurred even in low-density networks that were driven with spatially-restricted, correlated external stimuli. Hence, highly-simplified models when backed by a strong theoretical framework can be used to elucidate basic operating principles of networks. 


\section{Acknowledgement}

We thank B. Doiron, M. Long and M. Graupner for critical reading of the manuscript. JB was supported by a Human Frontier Science Program long-term postdoctoral fellowship (LT000132/2012) and by the Bettencourt Schueller Foundation.

\section{Author Contributions}

JB and AR designed the project. JB performed the experiments and analysed the results. JB and AR wrote the manuscript.

\section{Competing financial interests}

The authors declare no competing financial interests.

\section{References}

1 Deneve, S. \& Machens, C. K. Efficient codes and balanced networks. Nat Neurosci 19, 375-382, doi:10.1038/nn.4243 (2016).

2 Dichter, M. A. \& Ayala, G. F. Cellular mechanisms of epilepsy: a status report. Science 237, 157-164 (1987).

3 Trevelyan, A. J., Sussillo, D., Watson, B. O. \& Yuste, R. Modular propagation of epileptiform activity: evidence for an inhibitory veto in neocortex. J Neurosci 26, 12447-12455, doi:10.1523/JNEUROSCI.2787-06.2006 (2006).

4 Yizhar, O. et al. Neocortical excitation/inhibition balance in information processing and social dysfunction. Nature 477, 171-178, doi:10.1038/nature10360 (2011).

5 Rubenstein, J. L. \& Merzenich, M. M. Model of autism: increased ratio of excitation/inhibition in key neural systems. Genes Brain Behav 2, 255-267 (2003).

6 Lewis, D. A., Curley, A. A., Glausier, J. R. \& Volk, D. W. Cortical parvalbumin interneurons and cognitive dysfunction in schizophrenia. Trends Neurosci 35, 57-67, doi:10.1016/j.tins.2011.10.004 (2012).

7 van Vreeswijk, C. \& Sompolinsky, H. Chaos in neuronal networks with balanced excitatory and inhibitory activity. Science 274, 1724-1726 (1996).

8 van Vreeswijk, C. \& Sompolinsky, H. Chaotic balanced state in a model of cortical circuits. Neural Comput 10, 1321-1371 (1998). 
9 Gilman, J. P., Medalla, M. \& Luebke, J. I. Area-Specific Features of Pyramidal Neurons-a Comparative Study in Mouse and Rhesus Monkey. Cereb Cortex, doi:10.1093/cercor/bhw062 (2016).

10 DeNardo, L. A., Berns, D. S., DeLoach, K. \& Luo, L. Connectivity of mouse somatosensory and prefrontal cortex examined with trans-synaptic tracing. Nat Neurosci 18, 1687-1697, doi:10.1038/nn.4131 (2015).

11 Hooks, B. M. et al. Laminar analysis of excitatory local circuits in vibrissal motor and sensory cortical areas. PLoS Biol 9, e1000572, doi:10.1371/journal.pbio.1000572 (2011).

12 Bandeira, F., Lent, R. \& Herculano-Houzel, S. Changing numbers of neuronal and non-neuronal cells underlie postnatal brain growth in the rat. Proc Natl Acad Sci U S A 106, 14108-14113, doi:10.1073/pnas.0804650106 (2009).

13 Frick, A., Feldmeyer, D. \& Sakmann, B. Postnatal development of synaptic transmission in local networks of L5A pyramidal neurons in rat somatosensory cortex. J Physiol 585, 103-116, doi:10.1113/jphysiol.2007.141788 (2007).

14 Oswald, A. M. \& Reyes, A. D. Maturation of intrinsic and synaptic properties of layer 2/3 pyramidal neurons in mouse auditory cortex. J Neurophysiol 99, 2998-3008, doi:10.1152/jn.01160.2007 (2008).

15 Oswald, A. M. \& Reyes, A. D. Development of inhibitory timescales in auditory cortex. Cereb Cortex 21, 1351-1361, doi:10.1093/cercor/bhq214 (2011).

16 Citri, A. \& Malenka, R. C. Synaptic plasticity: multiple forms, functions, and mechanisms. Neuropsychopharmacology 33, 18-41, doi:10.1038/sj.npp.1301559 (2008).

17 Brunel, N. Dynamics of sparsely connected networks of excitatory and inhibitory spiking neurons. J Comput Neurosci 8, 183-208 (2000).

18 Markram, H. et al. Reconstruction and Simulation of Neocortical Microcircuitry. Cell 163, 456-492, doi:10.1016/j.cell.2015.09.029 (2015).

19 Goris, R. L., Movshon, J. A. \& Simoncelli, E. P. Partitioning neuronal variability. Nat Neurosci 17, 858-865, doi:10.1038/nn.3711 (2014).

20 Gur, M., Beylin, A. \& Snodderly, D. M. Response variability of neurons in primary visual cortex (V1) of alert monkeys. J Neurosci 17, 2914-2920 (1997).

21 Rust, N. C., Schultz, S. R. \& Movshon, J. A. A reciprocal relationship between reliability and responsiveness in developing visual cortical neurons. J Neurosci 22, 10519-10523 (2002).

22 Zohary, E., Shadlen, M. N. \& Newsome, W. T. Correlated neuronal discharge rate and its implications for psychophysical performance. Nature 370, 140-143, doi:10.1038/370140a0 (1994). 
23 Avermann, M., Tomm, C., Mateo, C., Gerstner, W. \& Petersen, C. C. Microcircuits of excitatory and inhibitory neurons in layer $2 / 3$ of mouse barrel cortex. $J$ Neurophysiol 107, 3116-3134, doi:10.1152/jn.00917.2011 (2012).

24 Levy, R. B. \& Reyes, A. D. Spatial profile of excitatory and inhibitory synaptic connectivity in mouse primary auditory cortex. J Neurosci 32, 5609-5619, doi:10.1523/JNEUROSCI.5158-11.2012 (2012).

25 Perin, R., Berger, T. K. \& Markram, H. A synaptic organizing principle for cortical neuronal groups. Proc Natl Acad Sci $U$ S A 108, 5419-5424, doi:10.1073/pnas.1016051108 (2011).

26 Pfeffer, C. K., Xue, M., He, M., Huang, Z. J. \& Scanziani, M. Inhibition of inhibition in visual cortex: the logic of connections between molecularly distinct interneurons. Nat Neurosci 16, 1068-1076, doi:10.1038/nn.3446 (2013).

27 Lefort, S., Tomm, C., Floyd Sarria, J. C. \& Petersen, C. C. The excitatory neuronal network of the $\mathrm{C} 2$ barrel column in mouse primary somatosensory cortex. Neuron $\mathbf{6 1}$, 301-316, doi:10.1016/j.neuron.2008.12.020 (2009).

28 Holmgren, C., Harkany, T., Svennenfors, B. \& Zilberter, Y. Pyramidal cell communication within local networks in layer $2 / 3$ of rat neocortex. $J$ Physiol 551, 139-153, doi:10.1113/jphysiol.2003.044784 (2003).

29 Markram, H., Lubke, J., Frotscher, M., Roth, A. \& Sakmann, B. Physiology and anatomy of synaptic connections between thick tufted pyramidal neurones in the developing rat neocortex. J Physiol 500 ( Pt 2), 409-440 (1997).

30 Renart, A. et al. The asynchronous state in cortical circuits. Science 327, 587-590, doi:10.1126/science.1179850 (2010).

31 de la Rocha, J., Doiron, B., Shea-Brown, E., Josic, K. \& Reyes, A. Correlation between neural spike trains increases with firing rate. Nature 448, 802-806, doi:10.1038/nature06028 (2007).

32 Gullo, F. et al. Orchestration of "presto" and "largo" synchrony in up-down activity of cortical networks. Front Neural Circuits 4, 11, doi:10.3389/fncir.2010.00011 (2010).

33 Soriano, J., Rodriguez Martinez, M., Tlusty, T. \& Moses, E. Development of input connections in neural cultures. Proc Natl Acad Sci U S A 105, 13758-13763, doi:10.1073/pnas.0707492105 (2008).

34 Wilson, N. R., Ty, M. T., Ingber, D. E., Sur, M. \& Liu, G. Synaptic reorganization in scaled networks of controlled size. $J$ Neurosci 27, 13581-13589, doi:10.1523/JNEUROSCI.3863-07.2007 (2007).

35 Ivenshitz, M. \& Segal, M. Neuronal density determines network connectivity and spontaneous activity in cultured hippocampus. J Neurophysiol 104, 1052-1060, doi:10.1152/jn.00914.2009 (2010).

36 Reyes, A. et al. Target-cell-specific facilitation and depression in neocortical circuits. Nat Neurosci 1, 279-285, doi:10.1038/1092 (1998). 
37 Roxin, A., Brunel, N., Hansel, D., Mongillo, G. \& van Vreeswijk, C. On the distribution of firing rates in networks of cortical neurons. J Neurosci 31, 1621716226, doi:10.1523/JNEUROSCI.1677-11.2011 (2011).

38 Buzsaki, G. \& Mizuseki, K. The log-dynamic brain: how skewed distributions affect network operations. Nat Rev Neurosci 15, 264-278, doi:10.1038/nrn3687 (2014).

39 Churchland, M. M. et al. Stimulus onset quenches neural variability: a widespread cortical phenomenon. Nat Neurosci 13, 369-378, doi:10.1038/nn.2501 (2010).

40 Litwin-Kumar, A. \& Doiron, B. Slow dynamics and high variability in balanced cortical networks with clustered connections. Nat Neurosci 15, 1498-1505, doi:10.1038/nn.3220 (2012).

41 Okun, M. \& Lampl, I. Instantaneous correlation of excitation and inhibition during ongoing and sensory-evoked activities. Nat Neurosci 11, 535-537, doi:10.1038/nn.2105 (2008).

42 Xue, M., Atallah, B. V. \& Scanziani, M. Equalizing excitation-inhibition ratios across visual cortical neurons. Nature 511, 596-600, doi:10.1038/nature13321 (2014).

43 Graupner, M. \& Reyes, A. D. Synaptic input correlations leading to membrane potential decorrelation of spontaneous activity in cortex. J Neurosci 33, 15075-15085, doi:10.1523/JNEUROSCI.0347-13.2013 (2013).

44 Turrigiano, G. G., Leslie, K. R., Desai, N. S., Rutherford, L. C. \& Nelson, S. B. Activity-dependent scaling of quantal amplitude in neocortical neurons. Nature 391, 892-896, doi:10.1038/36103 (1998).

45 Maffei, A., Nelson, S. B. \& Turrigiano, G. G. Selective reconfiguration of layer 4 visual cortical circuitry by visual deprivation. Nat Neurosci 7, 1353-1359, doi:10.1038/nn1351 (2004).

46 Ullian, E. M., Sapperstein, S. K., Christopherson, K. S. \& Barres, B. A. Control of synapse number by glia. Science 291, 657-661, doi:10.1126/science.291.5504.657 (2001).

47 Stellwagen, D. \& Malenka, R. C. Synaptic scaling mediated by glial TNF-alpha. Nature 440, 1054-1059, doi:10.1038/nature04671 (2006). 


\section{Figure Legends}
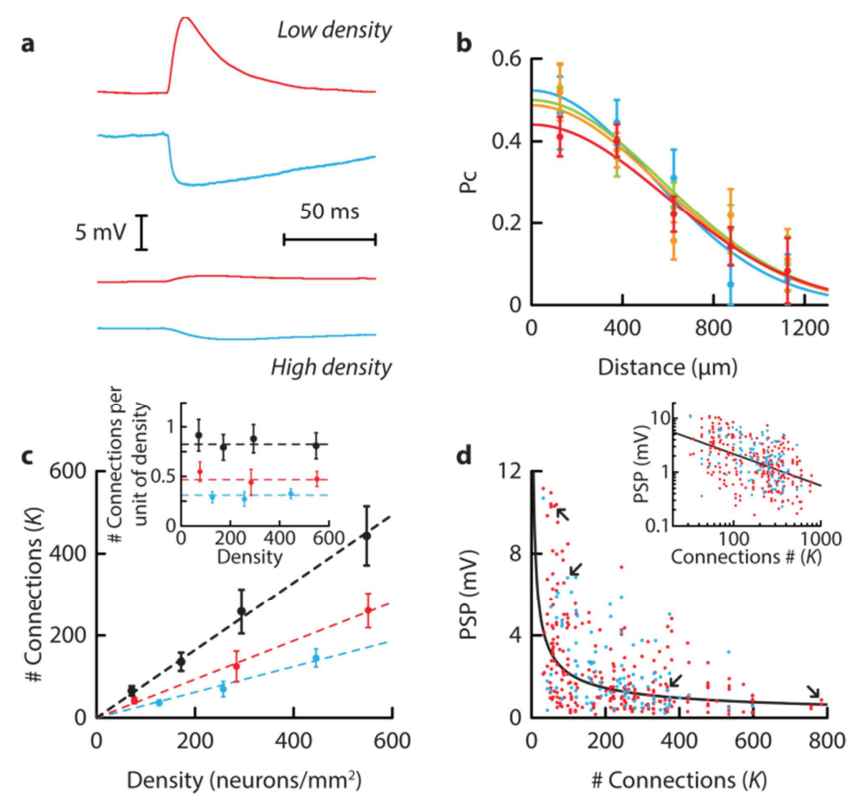

Figure 1: Synaptic scaling in networks of different sizes.

(a) Representative excitatory (red) and inhibitory (blue) PSPs in low and high density networks (arrows in d). (b) Connection probability vs intersomatic distance in networks with average densities of $72(\mathrm{n}=194$ connections tested in 25 preparations, blue), $171(\mathrm{n}=329$ in 45, green), $294\left(\mathrm{n}=270\right.$ in 32 , orange), $548(\mathrm{n}=287$ in 29 , red $)$ neurons $/ \mathrm{mm}^{2}$. All connection types are pooled together. Data presented as mean \pm SEM. (c) Number of connections $(K)$ vs density for E-to-E (red), I-to-E (blue) and total (black). Standard deviations calculated by bootstrapping data in $\mathbf{b}$. Inset: integral of the connection probability profile over space. (d) Amplitudes $(J)$ of unitary EPSPs (red, n = 261) and IPSPs (blue, $\mathrm{n}=99)$ vs. $K$. Inset: data in log-log scales. Slope of linear fit is -0.59 . 

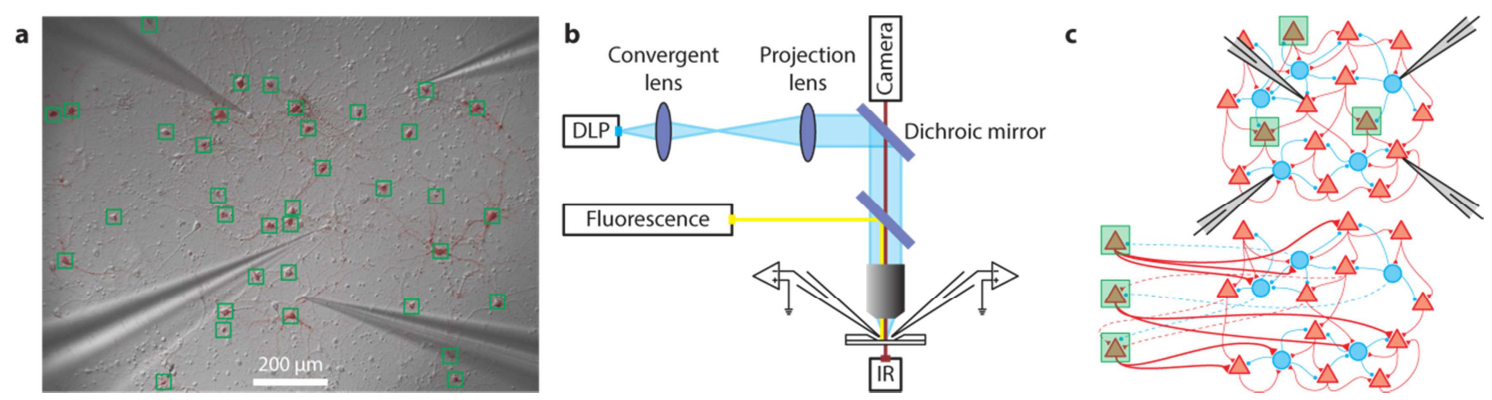

Figure 2: Optogenetic activation with spatio-temporal control.

(a) Culture visualized with IR-DIC and fluorescent microscopy. (b) Using a Digital Light Projector (DLP), the network was driven by delivering light pulses (green boxes in a) to neurons expressing ChR2 and a fluorescent tag (red). (c) The photostimulated ChR2 neurons (top) are effectively the external inputs to the network (bottom). 
a

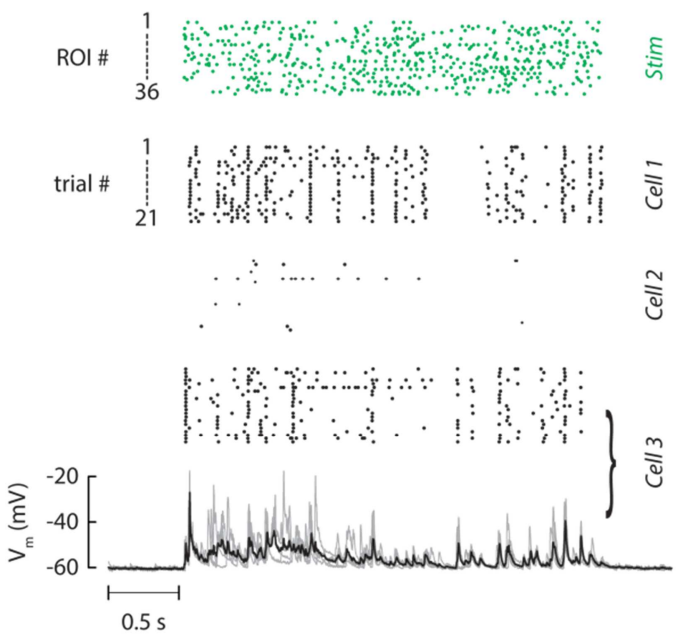

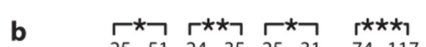

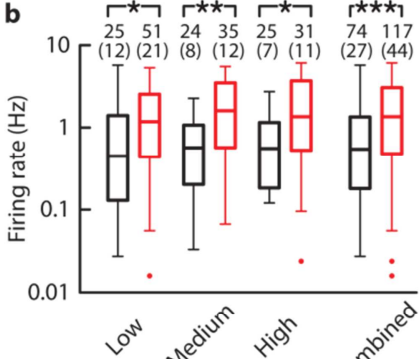

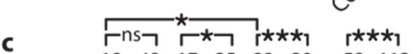

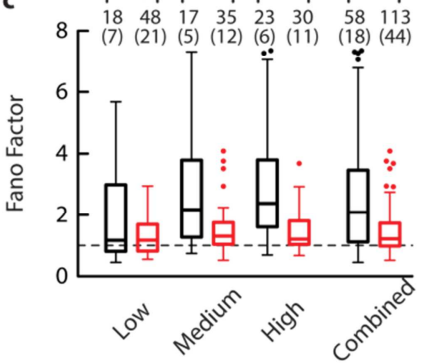

Figure 3: Evoked activity in the network.

(a) Top: raster plots showing the spatiotemporally-patterned stimuli (36 ROIs). Middle: rasters of evoked spikes (21 trials) in 3 simultaneously cell-attached recorded neurons. Bottom: whole-cell recordings from cell 3. Single trials (grey) superimposed on average (black). (b) Firing rate during spontaneous (black, average densities 97, 314, 744 neurons $/ \mathrm{mm}^{2}$ ) and evoked (red, average densities 188, 364, 653 neurons $/ \mathrm{mm}^{2}$ ) activity in networks of different densities. Also shown is the data combined across densities. Statistical significance was assessed using Mann-Whitney $U$-test (the number of recorded neurons is shown below the statistical significance bars and number of cultures in brackets). No statistical difference was found between densities for a given condition. (c) Fano factor of spike counts vs density during spontaneous (black; average densities 89, 289, 769 neurons $/ \mathrm{mm}^{2}$ ) and evoked (red; average densities 186, 364, 642 neurons $/ \mathrm{mm}^{2}$ ) activity (number of trials was 10-30). Box plots indicate median and interquartile range, whiskers cover the full range of the distribution and outliers are plotted individually. 

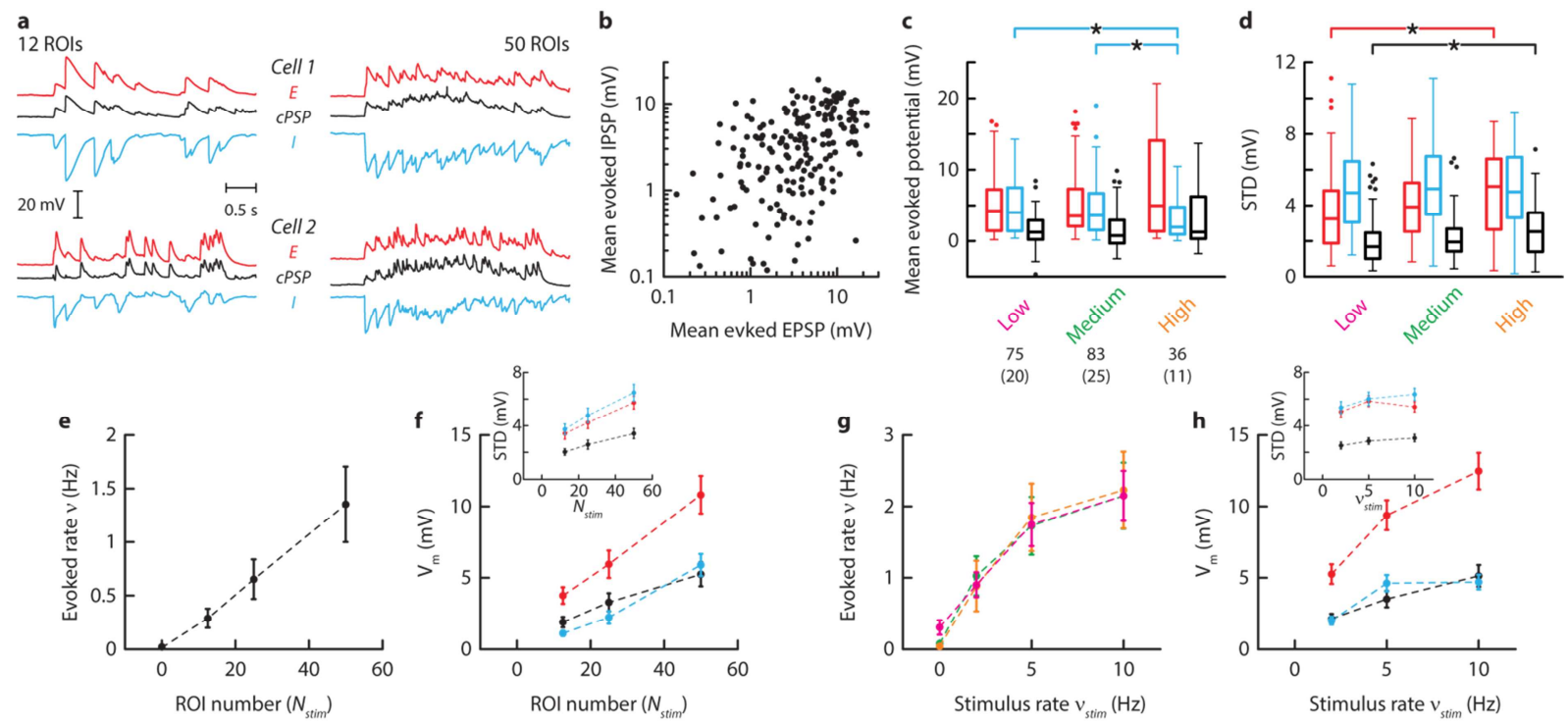

Figure 4: Excitation-inhibition balance in activated networks.

(a) Trial averaged isolated EPSPs (red) and IPSPs (blue), and composite PSPs (black) evoked with 12 \& 50 ROIs in 2 simultaneously recorded cells. (b) Plot of mean IPSP vs mean EPSP. Data points $(\mathrm{n}=194$ cells in 56 preparations) are average magnitudes during optical stimulation (input rate $v_{\text {stim }}=5 \mathrm{~Hz}$, stimulus correlation $C_{\text {stim }}=0$ ) relative to baseline. IPSP magnitude increased linearly with IPSP magnitude (Pearson correlation: $\mathrm{r}=0.45, p=3 \times 10^{-}$ $\left.{ }^{11}\right)$. (c) Average membrane potential deviation from baseline during stimulation for EPSPs (red), IPSPs (blue), and composite PSPs (black) in networks of different densities (116, 273, 544 neurons $/ \mathrm{mm}^{2}$ ). Only statistically significant tests using Mann-Whitney $U$-test are indicated. Neurons numbers are indicated below legends and numbers of preparations in brackets. Box plots indicate median and interquartile range, whiskers cover the full range of the distribution and outliers are plotted individually. (d) Same as in c but for the standard deviation of the membrane potential. (e) Firing rate vs ROI number ( $\mathrm{n}=16$ neurons in 5 preparations). (f) Average magnitude and standard deviation (inset) of EPSPs (red), IPSPs (blue), and composite PSPs (black) vs ROI number ( $\mathrm{n}=26$ neurons in 10 preparations). (g) Evoked firing rate vs stimulus rate of light pulses delivered to each ROI in low $(n=20$ neurons in 8 preparations, magenta), medium ( $\mathrm{n}=22$ neurons in 6 preparations, green), and high ( $\mathrm{n}=18$ in 6 preparations, orange) density networks. (h) Average magnitudes and standard deviation (inset) of EPSPs (red), IPSPs (blue), and composite PSPs (black) vs stimulus rate $(\mathrm{n}=45$ neurons in 12 preparations). Data presented as mean \pm SEM. 


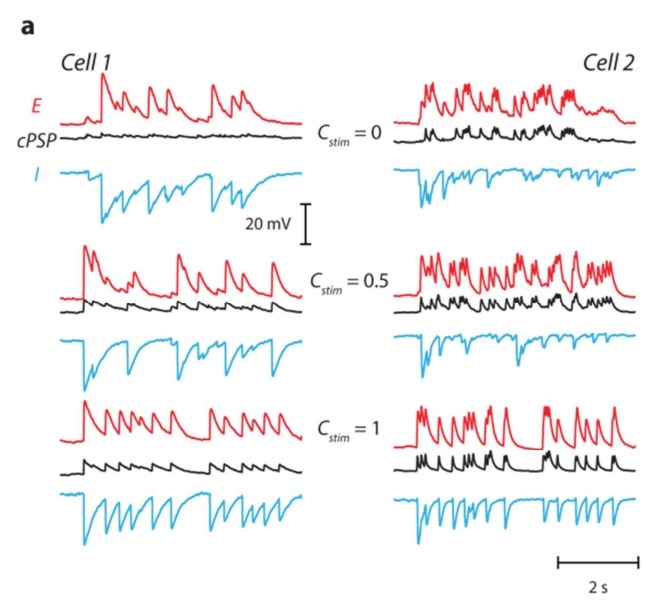

b
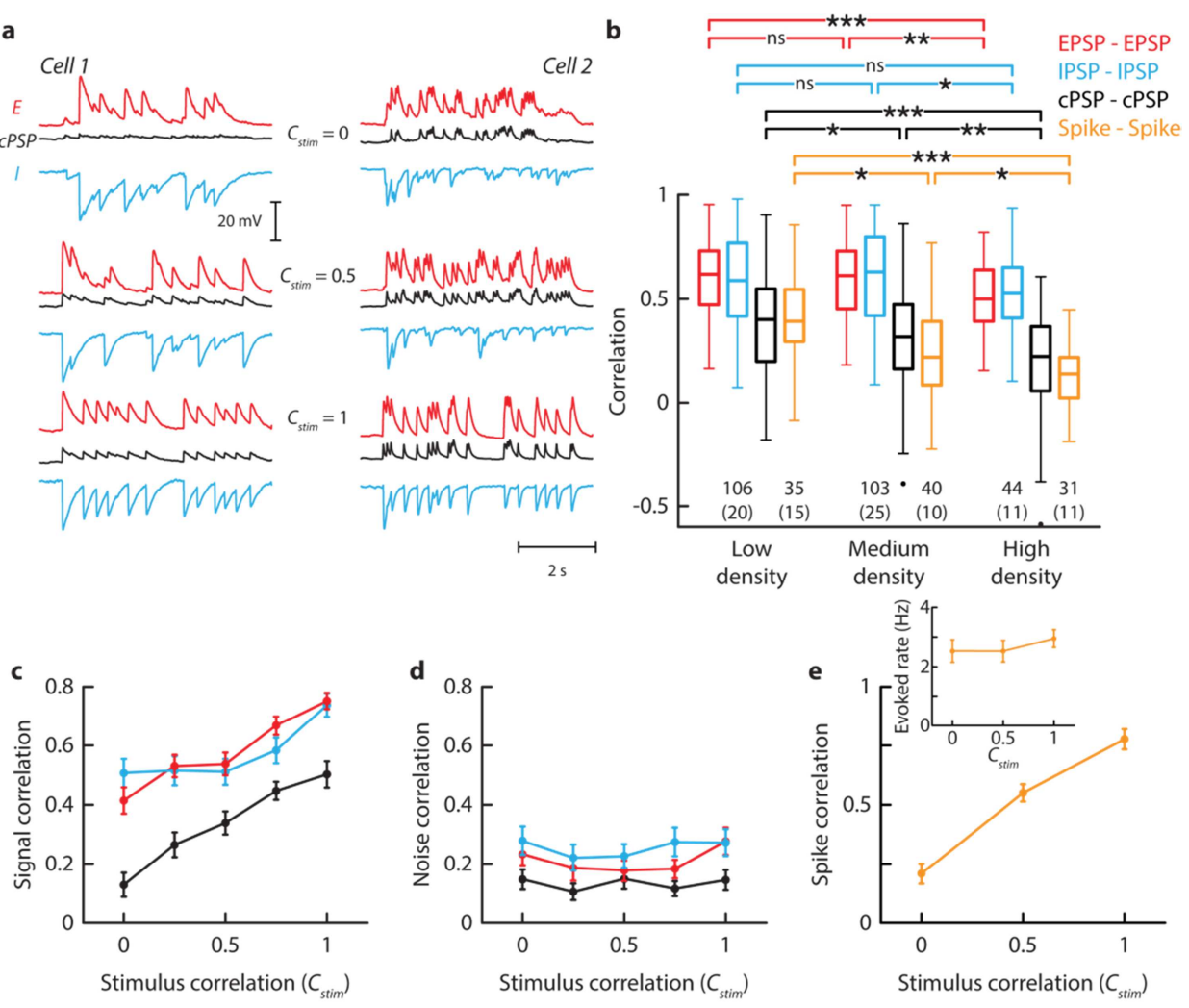

Figure 5: Correlations in activated networks.

(a) Trial averaged membrane potentials for two simultaneously recorded cells when the correlations between ROIs ( $\left.C_{\text {stim }}\right)$ were 0 (top), 0.5 (middle), and 1 (bottom). (b) Excitatory (red), inhibitory (blue), composite PSP (grey), and spike (orange) zero-lag correlation coefficient vs density. Stimulus rate $v_{\text {stim }}=5 \mathrm{~Hz}$ and correlation $C_{\text {stim }}=0$. For membrane potential average densities were 116, 273, 544 neurons $/ \mathrm{mm}^{2}$ and for spikes average densities were 199, 374, 653 neurons $/ \mathrm{mm}^{2}$. Statistical significance was assessed using Mann-Whitney $U$-test. Numbers of neuronal pairs are indicated below whisker plots (box: median and interquartile range, whiskers: full range of the distribution, outliers are plotted individually) and numbers of preparations in brackets. (c, d) Correlations between isolated EPSPs (red), isolated IPSPs (blue) and composite PSPs (black) for signal (d) and noise (e) correlations ( $\mathrm{n}=45$ pairs in 12 preparations). (e) Spike correlation vs $C_{\text {stim }}(\mathrm{n}=28$ pairs in 8 preparations). Inset: corresponding evoked firing rate. 

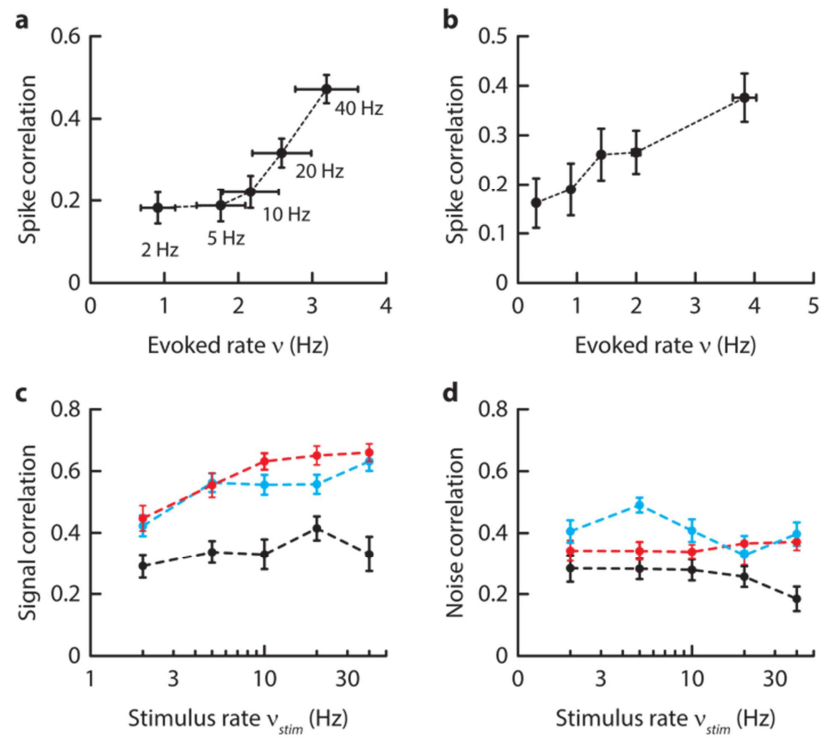

Figure 6: Relationship between rate and correlation.

(a) Plot of spike correlation vs geometric mean of the firing rates evoked by different stimulus rate $v_{\text {stim }}\left(\mathrm{n}=37\right.$ pairs in 9 preparations). Numbers indicate $v_{\text {stim }}$. (b) Spike correlation vs geometric mean of the firing rates (constant $v_{\text {stim }}=5 \mathrm{~Hz}$; data were pooled according to their mean firing rates; each data point represents $\sim 21$ pairs, in total 106 neuronal pairs in 44 preparations). (c, d) Signal (c) and noise (d) correlation vs $v_{\text {stim }}$ for EPSPs (red), IPSPs (blue), and cPSPs (black) ( $\mathrm{n}=48$ neuronal pairs in 9 preparations; $C_{\text {stim }}=0$; 30-60 ROIs). Data presented as mean $\pm \mathrm{SEM}$. 


\section{Methods}

\section{Primary neuron cultures and expression of channelrhodopsin}

Dissociated cortical neurons from postnatal (P0-P1) mice were prepared as described previously ${ }^{48}$ and in accordance with guidelines of the New York University Animal Welfare Committee. Briefly, the mouse cortex was dissected in cold CMF-HBSS $\left(\mathrm{Ca}^{2+}\right.$ and $\mathrm{Mg}^{2+}$ free Hank's balanced salt solution containing $1 \mathrm{mM}$ pyruvate, $15 \mathrm{mM}$ HEPES, $10 \mathrm{mM} \mathrm{NaHCO} 3$ ). The tissue was dissociated in papain ( $15 \mathrm{U} / \mathrm{mL}$, Roche) containing $1 \mathrm{mM}$ L-cystein, $5 \mathrm{mM} 2$ amino-5-phosphonopentanoic acid and $100 \mathrm{U} / \mathrm{ml}$ DNase (DN25; Sigma) for $25 \mathrm{~min}$. After enzymatic inactivation in CMF-HBSS containing $100 \mathrm{mg} / \mathrm{mL}$ BSA (A9418; Sigma) and $40 \mathrm{mg} / \mathrm{mL}$ trypsin inhibitor (T9253; Sigma), pieces were mechanically dissociated with a pipette. Cells concentration was measured before plating using a haemocytometer. Approximately $0.2-3 \times 10^{6}$ cells were plated on each coverslip, resulting in a density of $\sim 50$ 1,000 cells $/ \mathrm{mm}^{2}$ at the time of experiment. Neurons were seeded onto German glass coverslips (25 mm, \#1 thickness, Electron Microscopy Science). Glass was cleaned in $3 \mathrm{~N}$ $\mathrm{HCl}$ for $48 \mathrm{~h}$ and immersed in sterile aqueous solution of $0.1 \mathrm{mg} / \mathrm{mL}$ poly-L-lysine (MW: 70,000 - 150,000; Sigma) in 0.1 M borate buffer for $12 \mathrm{~h}$. Neurons were grown in Neurobasal medium (supplemented with B27, Glutamax and penicillin/streptomycin cocktail; Invitrogen) in a humidified incubator at $37^{\circ} \mathrm{C}, 5 \% \mathrm{CO}_{2}$. One third of the culture medium was exchanged every 3 days.

Expression of channelrhodopsin (ChR2) in excitatory neurons was achieved by crossing homozygote Vglut2-Cre mice (016963, Jackson Laboratory) with ChR2-loxP mice (Ai32, 012569, Jackson Laboratory). Alternatively, ChR2 expression was achieved by viral infection with AAV2-hSyn-hChR2(H134R)-mCherry of cortical neurons harvested from Swiss Webster wild type mice (Jackson Laboratory). The virus was produced at $3 \times 10^{12} \mathrm{cfu} / \mathrm{mL}$ by the University of North Carolina Vector Core Services using plasmid provided by Karl Deisseroth (Stanford University). At 3 days in vitro (DIV), the culture was infected with $1 \mu \mathrm{L}$ of virus. Experiments were performed at 14-21 DIV, when neuronal characteristics and network connectivity were stable and expression of ChR2 was sufficient to enable reliable photostimulation.

\section{Electrophysiological recordings}


Recordings were performed at room temperature in artificial cerebrospinal fluid (aCSF) bubbled with $95 \% \mathrm{O}_{2}$ and $5 \% \mathrm{CO}_{2}$. The aCSF solution contained (in $\mathrm{mM}$ ): $125 \mathrm{NaCl}, 25$ $\mathrm{NaHCO}_{3}, 25 \mathrm{D}$-glucose, $2.5 \mathrm{KCl}, 2 \mathrm{CaCl}_{2}, 1.25 \mathrm{NaH}_{2} \mathrm{PO}_{4}$ and $1 \mathrm{MgCl}_{2}$. An alternative aCSF solution, in which $10 \mathrm{mM}$ HEPES replaces the equivalent concentration of $\mathrm{NaHCO}_{3}$, was also used to avoid perfusion during the experiment. Electrodes, pulled from borosilicate pipettes (1.5 OD) on a Flaming/Brown micropipette puller (Sutter Instruments), had resistances in the range of 6-10 $\mathrm{M} \Omega$ when filled with internal solution containing (in $\mathrm{mM}$ ): $130 \mathrm{~K}$-gluconate, 10 HEPES, 10 phosphocreatine, $5 \mathrm{KCl}, 1 \mathrm{MgCl}_{2}, 4$ ATP-Mg and $0.3 \mathrm{mM}$ GTP. In some experiments, $5 \mathrm{mM}$ of QX-314 was added to block the action potentials internally.

Cells were visualized through a $\times 10$ water-immersion objective using infrared differential interference contrast (IR-DIC) and fluorescence microscopy (BX51, Olympus). Simultaneous whole-cell current-clamp recordings were made from up to four neurons using BVC-700A amplifiers (Dagan). The signal was filtered at $5 \mathrm{kHz}$ and digitized at $25 \mathrm{kHz}$ using an 18-bits interface card (PCI-6289, National Instrument). Signal generation and acquisition were here and in the following controlled by a custom user interface programmed with LabVIEW (National Instrument).

\section{Analysis of intrinsic and network properties of cortical cultures}

For every experiment, IR-DIC images around the region of recording were saved for off-line examination using a custom user interface programmed with LabVIEW (National Instrument). Neuronal cultures can have local variations in their densities. Thus, cell density was determined locally by counting somata on $\mathrm{a} \sim 1 \times 1 \mathrm{~mm}^{2}$ area around the recording site. In Figs. 3b-c, 4c-d, 4g (and Supplementary Fig. 9a), and 5b, data were pooled according to densities. Low, medium and high densities corresponded to networks of neuronal densities (in neurons $/ \mathrm{mm}^{2}$ ): density $<200,200<$ density $<450$, and $450<$ density, respectively.

Intrinsic properties (Supplementary Table 1 and Supplementary Fig. 1) were characterized by applying 15 current steps (-0.1 to $0.5 \mathrm{nA})$. The subthreshold membrane (input resistance, membrane time constant) and suprathreshold firing (spike threshold, spike width, afterhyperpolarization, rheobase, maximum firing rate) were measured using standard protocols programmed in Matlab (Mathworks).

To characterize post-synaptic potentials (PSPs), paired recordings were made from two neurons. Brief (20 ms; 0.1-0.4 nA) suprathreshold current pulses were delivered to one cell 
and the PSP (if connected) was measured in the other cell. The PSP parameters ( $E$ or $I$, magnitude, time-to-peak) were documented (Supplementary Table 2). By performing many paired recordings and documenting the distance between cell somata, the connection probability $\left(P_{c}=\right.$ number of connections / total tested) spatial profile (Supplementary Fig. 2) could be determined. The data points were grouped into $250 \mu \mathrm{m}$ bins and the spatial profile fitted with a Gaussian function:

$$
P_{c}(x)=p_{0} \cdot \exp \left(-x^{2} / 2 \sigma^{2}\right)
$$

where $p_{0}$ is peak probability, $\sigma$ represents the spread of connectivity, and $x$ is the distance from the centre.

In Fig. 1b, data were first pooled according to density to compute the connection profile for each density. Then, the number of connections $K$ plotted in Fig. 1c was calculated by integration. The number of neurons contained in the annulus between $x$ and $x+d x$ is:

$$
N(x)=\operatorname{density} \cdot 2 \pi x \cdot d x
$$

The number of connections is then computed by summing the number of connected neurons in this annulus over space:

$$
K=\int_{0}^{+\infty} N(x) \cdot P_{c}(x)=\text { density } \cdot \sigma^{2} 2 \pi p_{0}
$$

Note that we used infinite boundaries that might appear unrealistic because axons have finite lengths. However the Gaussian shape of the connection profile assures that the integral rapidly converges such that the contribution of long distance connections is minimal (for example, limiting the integration to $x=1,500 \mu \mathrm{m}$ reduced $K$ by only $5 \%$ ).

We used a bootstrap procedure to estimate the standard deviation of calculated $K$. For each density, we randomly subsampled $50 \%$ of the data, fitted the resulting profile as above (Eq. 1), and computed the expected number of connections (Eq. 3). This procedure was repeated 200 times to obtain an estimate for the mean and the standard deviation of the distribution (Fig. 1c).

\section{Optical stimulation setup}


A Digital Light Processing projector (DLP LightCrafter; Texas Instrument) was used to stimulate optically neurons expressing ChR2. The projector had a resolution of $608 \times 684$ pixels. The image of the projector was demagnified and collimated using a pair of achromatic doublet lenses (35 mm and $200 \mathrm{~mm}$; Thorlabs; Fig. 2b of main text). A dual port intermediate unit (U-DP, Olympus), containing a $510 \mathrm{~nm}$ dichroic mirror (T510LPXRXT, Chroma), was placed between the fluorescent port and the projection lens. The resulting pixel size at the sample plane was a rectangle of dimensions $2.2 \mu \mathrm{m} \times 1.1 \mu \mathrm{m}$. The blue LED of the projector, with $460 \mathrm{~nm}$ centre wavelength, was used to stimulate the ChR2-expressing neurons.

The CCD camera (C8484, Hamamatsu) was used to calibrate the light intensity as follows. The short-pass dichroic mirror was replaced with a half reflecting mirror and a $100 \%$ reflecting mirror was placed at the sample plane. This allowed measurement of the light intensity at the point of stimulation and at other regions to estimate the contrast ratio. This is an important measure because it provides an estimate of the light intensity that a nonstimulated neuron receives during stimulation of the ChR2 expressing neurons. There are two common methods of measuring contrast. The full-on full-off method measures the averaged brightness of a white and a black test pattern and expresses the two measurements as a ratio of white to black. The measured ratio was $815: 1$, close to the $685: 1$ ratio specified by the manufacturer. The ANSI contrast measurement uses a checkerboard pattern composed of 16 rectangles, eight white and eight black. The contrast ratio is then defined as the quotient of the averaged white pixels to the averaged black pixels. The ANSI contrast was 21:1 (compared to the 43:1 value provided by the manufacturer), which gives a lower bound for the contrast ratio. To get an insight of the real contrast ratio during experiment, namely to measure background illumination of our system, we measured the contrast ratio when a single region of interest was illuminated or when 20 areas were simultaneously illuminated. We found the respective values of 700:1 and 170:1. During experiments, we used a light intensity of $10 \mathrm{~mW} / \mathrm{mm}^{2}$, which was calibrated with the camera and confirmed with a light power meter. Thus, we estimated the background light intensity to lie between 10 and $50 \mu \mathrm{W} / \mathrm{mm}^{2}$ during photostimulation, which would give rise to photocurrent of about 8-32 pA. This value, while insufficient to elicit spikes, could nevertheless generate postsynaptic potentials on the order of 0.1 to $1 \mathrm{mV}$. We therefore recorded neurons that either did not express ChR2 or whose dendritic processes did not overlap with the stimulated cells to avoid any spurious correlation with the stimulus. However, note that the largest estimate of background stimulation is only relevant when neurons are synchronously activated. 
Images were streamed continuously at a rate of $1,440 \mathrm{~Hz}$ from the computer to the projector via a graphic card (01G-P3-1526-KR; EVGA) using the HDMI port. We also measured the light intensity at the output of the projector using a photodiode (TSL13T; Texas Advanced Optoelectronics Solutions Inc.). We used this signal as an accurate trigger for synchronizing photostimulation and electrophysiological recordings offline.

\section{Stimulation and recordings protocols}

The cultured network was optically stimulated as follows. After identifying the fluorescent ChR2-expressing neurons, a subset of these neurons were designated for photo-stimulation with regions of interest or ROIs that surrounded their somata (Fig. 2a). Typically, 15-60 ROIs were used so that about $10 \%$ of neurons in the field were stimulated. A (3-5 seconds) train of brief light pulses $(5 \mathrm{~ms})$ was delivered to the neurons in each ROI.

Although the sequence of light pulses could be generated using a Poisson process, we opted to use noise-driven leaky-integrate-and-fire (LIF) neuron models instead. With the appropriate parameters, the use of LIF minimized the occurrences of closely-spaced spikes, which would cause failure in activation of ChR2 (see Supplementary Fig. 5). The LIF model had a membrane time constant $\tau_{m}=60 \mathrm{~ms}$ and input resistance $R_{m}=300 \mathrm{M} \Omega$. The input current to each unit was a sum of a time varying $I_{k}(t)$ and a constant $I_{c s t}$ component so that $V_{k}(t)$ obeys:

$$
\tau_{m} \dot{V}_{k}(t)=-V_{k}(t)+R_{m}\left(I_{c s t}+I_{k}(t)\right)
$$

A spike was generated when $V_{k}$ exceeded the voltage threshold $V_{t}=-50 \mathrm{mV}$ and was then reset to $V_{\text {reset }}=-65 \mathrm{mV}$. The initial condition was $V_{k}\left(t_{0}\right)=-60 \mathrm{mV}$. Given the parameters mentioned here and the statistics of the noisy current input $I_{k}(t)$ (see below), the generated spike trains had an average firing rate $v_{\text {stim }} \approx 4.8 \mathrm{~Hz}$ and a Fano factor of $\sim 1$. The large membrane time constant and the refractory period $t_{\text {ref }}=10 \mathrm{~ms}$ eliminated high frequency bursts. The stimulus rate $v_{\text {stim }}$ was modulated by varying the constant input current $I_{c s t}$.

The input current obeys the following equation (see Supplementary Fig. 4):

$$
I_{k}(t)=\sqrt{C_{\text {stim }}} \cdot I_{\text {com }}(t)+\sqrt{1-C_{\text {stim }}} \cdot I_{\text {ind }, k}(t)
$$


where $I_{c o m}(t)$ is a noisy input common to all stimulated neurons, $I_{i n d, k}(t)$ is an independent noisy input to each neuron $k$, and $C_{\text {stim }}$ is a constant. Because $I_{\text {com }}(t)$ and $I_{\text {ind,k }}(t)$ have the same variance and are independent random variables, the cross-correlation coefficient between two input currents $I_{k}(t)$ and $I_{l}(t)$ converges to $C_{\text {stim }}$ for long time series:

$$
C_{s t i m}=\frac{\left\langle I_{k}(t) \cdot I_{l}(t)\right\rangle_{t}}{\sqrt{\left\langle I_{k}(t)^{2}\right\rangle_{t} \cdot\left\langle I_{l}(t)^{2}\right\rangle_{t}}}
$$

where $\langle\cdot\rangle_{t}$ denotes average over time. The spatial cross-correlation $C_{\text {stim }}$ (called here "stimulus correlation") was varied between 0 and 1 (in steps of 0.25). The resulting spike correlation measured as the Pearson correlation of the firing rates also varies between 0 and 1 (see Supplementary Fig. 4b). However, because this measure depends on the count window, we used the current correlation $C_{\text {stim }}$ to refer to stimulus correlation in the main text.

$I_{\text {com }}(t)$ and $I_{\text {ind,k }}(t)$ were realizations of a Gaussian (Ornstein-Uhlenbeck) noise process and were generated using:

$$
I\left(t_{n+1}\right)=I\left(t_{n}\right) \cdot e^{-d t / \tau}+\sigma \cdot \sqrt{1-e^{-2 d t / \tau}} \cdot \xi\left(t_{n}\right)
$$

where $I\left(t_{0}\right)=0$ is the initial condition, $\sigma=24 \mathrm{pA}$ is the standard deviation, $\tau=50 \mathrm{~ms}$ is the correlation time and $\xi\left(t_{n}\right)$ is a random variable drawn from the standard normal distribution of zero mean and unity variance.

During data collection, the stimuli (with specified $v_{\text {stim }}$ and $C_{\text {stim }}$ ) were delivered 5-6 times if subthreshold potentials were recorded in whole-cell mode and 10-30 times if spikes were recorded in cell-attached mode. At least $5 \mathrm{~s}$ separated each stimulation.

\section{Data Analyses}

As observed in other in vitro studies in cultures ${ }^{49}$ or in slices ${ }^{43}$, spontaneous network-wide bursts occurred. Spontaneous, correlated bursts have also been observed in vivo ${ }^{50-52}$ indicating that they are not artefacts of the in vitro preparation. Under the conditions of the experiments, these bursts were infrequent ( 1-2 bursts/minute) and were readily identified based on their long duration ( $\gtrsim 1 \mathrm{sec}$ ) high frequency spikes or depolarization, and by the fact that they were observed simultaneously in all the recording electrodes. These events were 
excluded from the analysis as they represented a different activity regime ${ }^{43}$ of debated origin (e.g. refs ${ }^{53-55}$ ). The theoretical implications of these bursts are beyond the scope of the paper and are currently under active investigation.

\section{$\underline{\text { Analysis of spike data }}$}

The spike rate was defined as the number of spikes divided by the stimulation period. To estimate spike correlation, spikes were first binned at $d t=1 \mathrm{~ms}$. The firing rate $r_{i}^{n}(t)$ of neuron $i$ for the $\mathrm{n} t h$ realization $\left(n \in\left\{1 . . N_{\text {trials }}\right\}\right.$ ) was computed by convolving the spike count with a Gaussian kernel of width $\Delta t=50 \mathrm{~ms}$. The average firing rate $\tilde{r}_{i}(t)$ over the various trials of the same stimulation protocol was defined as: $\tilde{r}_{i}(t)=\left\langle r_{i}^{n}(t)\right\rangle_{n}$, where $\langle\cdot\rangle_{n}$ denotes average over trials.

The spike correlation coefficient between neuron $i$ and neuron $j$ was estimated as the Pearson coefficient between $\tilde{r}_{i}(t)$ and $\tilde{r}_{j}(t)$ during the stimulation period. The spike count Fano factor was calculated as the variance of spike count in a $500 \mathrm{~ms}$ time window (or other lengths; Supplementary Fig. 8) divided by the mean. This quantity was computed across trials before being averaged over time. Very low firing neurons (rate $<0.1 \mathrm{~Hz}$ ) were excluded of this analysis.

\section{Analysis of membrane potential data}

The membrane potential $V$ of neuron $i$ for the $n t h$ realization $\left(n \in\left\{1 \ldots N_{\text {trials }}\right\}\right)$ is denoted as: $V_{i}^{n}(t)$. We define signal as the average response $\tilde{V}_{i}(t)$ over the various trials of the same stimulation protocol:

$$
\tilde{V}_{i}(t)=\left\langle V_{i}^{n}(t)\right\rangle_{n}
$$

The average depolarization (or hyperpolarization) was defined as the difference between the mean membrane potential during external stimulation and its value at rest (Figs. 4c, 4f, 4h, Supplementary Figs. 9, 11, and 12). The standard deviation during evoked activity was measured as the standard deviation of the membrane potential computed during the stimulation period (Figs. 4d, 4f, 4h, Supplementary Figs. 9, 11, and 12). This value was then averaged across trials. 
We calculated the zero-lag correlation coefficient between membrane potentials of neurons recorded simultaneously (Figs. 5b-d, 6c-d, Supplementary Fig. 12). We defined the signal correlation as the correlation between the membrane potentials $\tilde{V}_{i}(t)$ of neuron 1 and neuron 2 averaged over trials as:

$$
r_{V_{1}, V_{2}}^{\text {signal }}=\frac{\left\langle\left(\tilde{V}_{1}(t)-\overline{\tilde{V}}_{1}\right) \cdot\left(\tilde{V}_{2}(t)-\overline{\tilde{V}}_{2}\right)\right\rangle_{t}}{\sqrt{\sigma_{\widetilde{V}_{1}} \sigma_{\widetilde{V}_{2}}}}
$$

where $\bar{V}_{l}=\left\langle\tilde{V}_{i}(t)\right\rangle_{t}$ is the average over time.

Additionally, we defined here the noise response of neuron $i$ as the deviation of a given trial $n$ from the mean response:

$$
V_{\text {noise }, i}^{n}(t)=V_{i}^{n}(t)-\left\langle V_{i}^{n}(t)\right\rangle_{n}
$$

Similarly, the noise correlation between neurons 1 and neuron 2 is defined by the following:

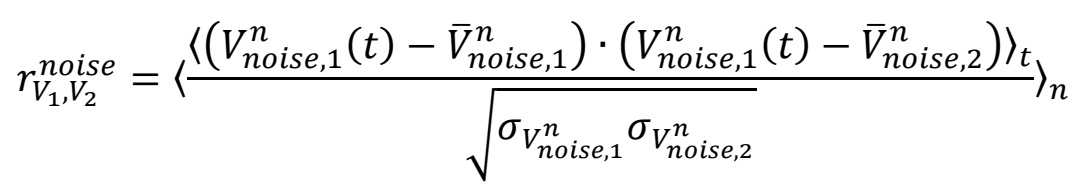

Noise correlation was thus computed for each trial and then averaged across trials.

\section{$\underline{\text { Statistical analysis }}$}

Data collection and analysis were not performed blind to the conditions of the experiments. No randomization method was used to collect data and no data point or animal were excluded. All the data were shown as mean \pm SEM., unless stated otherwise. Two group comparisons were performed using either paired or unpaired two-sided Mann-Whitney $U$-test. Firing rates which had a long tail distribution were first log transformed before being compared. A generalized linear regression model with Bonferroni correction was performed when more than two groups were compared. The variances between groups were assumed to be different. No statistical methods were used to pre-determine sample sizes.

\section{$\underline{\text { Data availability }}$}


The data that support the findings of this study are available from the corresponding author upon request.

\section{Code availability}

Data acquisition (Labview) and analysis (Labview or Matlab) software used in this paper are described in the Methods and will be available upon request.

\section{Methods-only References}

48 Hilgenberg, L. G. \& Smith, M. A. Preparation of dissociated mouse cortical neuron cultures. J Vis Exp, 562, doi:10.3791/562 (2007).

49 Wagenaar, D. A., Pine, J. \& Potter, S. M. An extremely rich repertoire of bursting patterns during the development of cortical cultures. BMC Neurosci 7, 11, doi:10.1186/1471-2202-7-11 (2006).

50 Leinekugel, X. et al. Correlated bursts of activity in the neonatal hippocampus in vivo. Science 296, 2049-2052, doi:10.1126/science.1071111 (2002).

51 Chiu, C. \& Weliky, M. Spontaneous activity in developing ferret visual cortex in vivo. J Neurosci 21, 8906-8914 (2001).

52 Weliky, M. \& Katz, L. C. Correlational structure of spontaneous neuronal activity in the developing lateral geniculate nucleus in vivo. Science 285, 599-604 (1999).

53 Eytan, D. \& Marom, S. Dynamics and effective topology underlying synchronization in networks of cortical neurons. $J$ Neurosci 26, 8465-8476, doi:10.1523/JNEUROSCI.1627-06.2006 (2006).

54 Orlandi, J. G., Soriano, J., Alvarez-Lacalle, E., Teller, S. \& Casademunt, J. Noise focusing and the emergence of coherent activity in neuronal cultures. Nature Physics 9, 582-590, doi:10.1038/nphys2686 (2013).

55 Feinerman, O., Segal, M. \& Moses, E. Identification and dynamics of spontaneous burst initiation zones in unidimensional neuronal cultures. J Neurophysiol 97, 29372948, doi:10.1152/jn.00958.2006 (2007). 